\title{
'FROM OUTCAST TO INBOARD': THE TRANSMISSION, PROFESSIONALISATION AND INTEGRATION OF ACUPUNCTURE INTO BRITISH MEDICAL CULTURE
}

\author{
SuZANNE TANG
}

\begin{abstract}
Early forms of body piercing to move the body's $q i$ have their social and cultural origins over 2,000 years ago in China, thousands of miles away from British soil, where today modern Chinese acupuncture is used by approximately 16 per cent of the public ${ }^{2}$ and 37 per cent of primary-care practices. ${ }^{3}$ This process of cross-cultural assimilation has taken place over a number of phases, spanning over 400 years; and has been a result of interested individuals and particular historical occurrences. Today, acupuncture is at the forefront of the state regulation of Complementary and Alternative Medicine (CAM), which seems to illustrates just how far acupuncture has integrated into British medical culture. However, a closer look at this debate reveals not only serious flaws in the processes of professionalisation and integration of acupuncture, but it also raises significant doubts as to their merits.
\end{abstract}

\section{Early transmission of acupuncture: the seventeenth to early twentieth centuries}

Information about acupuncture was transmitted to Europe and America in three distinct phases. ${ }^{4}$ As early as the seventeenth century, Jesuits and surgeons working with the European trading companies, in particular the Dutch East India Company, first came into contact with acupuncture practices of China and Japan and wrote several treatises describing the technique. The second phase took place during the eighteenth and nineteenth centuries, during which Chinese and Japanese texts were translated by scholars and doctors,

1 Dowie 2003, p. 24.

${ }^{2}$ Fisher and Ward 1994, p. 107.

3 Abdulla 1998, p. 962.

4 As outlined in Bivins 2000, pp. 1-14.

(C) Brill, Leiden, 2006

ASME 2,2 
particularly those in France, who then attempted to assimilate them into the 'new science' of contemporary Western medical knowledge, such as Galvanism, Mesmerism and clinical medicine. Finally, in the twentieth century, acupuncture made a resurgence that managed to secure its survival in the West until today. These early phases of the transmission process were largely dependent on a handful of individuals, whose interest and enthusiasm led to several publications that awakened Europeans to the Asian practice and stirred many debates within the academic, medical and lay spheres. It was also through their translations and descriptions of acupuncture in the East that it came to be defined by the physical and materialistic application of the needling technique. The theoretical framework underlying its practice became, for the most part, misunderstood or disregarded because its language and philosophy were irreconcilable with western scientific thought. Wilhelm Ten Rhyne, Engelbert Kaempfer, James Morss Churchill and George Souille de Morant were some of the key figures who promoted the use of acupuncture in Europe during the seventeenth to the early twentieth centuries, and together with the thread of orientalism and periods of intense medical debate, enabled the practice to gain a foothold, albeit a loose one, in the dominant local medical culture. ${ }^{5}$

\section{The twentieth-century acupuncture: $1950-1990$ s}

In the late twentieth century, acupuncture enjoyed a renaissance and was finally able to at least compete with the technologically developed and empirically based modern standardised medicine, to receive lasting affirmation from broad sectors of the population. In addition, similar to the earlier phases of assimilation, this resurgence was also embedded within certain historical events and movements, such as the Communist campaign that constructed Traditional Chinese Medicine (TCM), the opening up of China to the West and the alternative medicine movement that played significant roles in creating an environment that helped to propel acupuncture to a new level of popularity and medical interest.

${ }^{5}$ See Ten Rhyne 1683; Kaempfer 1996; Churchill 1822; and Souille de Morant 1939-1941. 


\section{Construction of TCM}

Western medicine began to dominate the Chinese medical market in the early decades of the twentieth century. Subsequently traditional medicine lost its status and its practitioners feared for the continuation of their craft. However, during the civil war with the Nationalist party, Chinese medicine became an integral part of the Communist campaign under Mao Zedong's guidance, who allied himself with the ostracised members of society, such as the Chinese medical practitioners and branded Western medicine bourgeois. After the establishment of the People's Republic of China in 1949, Chinese medicine was named a 'Great treasure-house'6 and its development became 'inseparably intertwined with the establishment of the new China'.? The strategy to embrace and promote Chinese medicine included raising the subordinate status of Chinese medicine to equal that of Western medicine in a United Medicine of China through the establishment of the Research Academies of Traditional Chinese Medicine (TCM) in 1954, and later the Academies or Colleges of TCM in 1956, which institutionalised and standardised traditional Chinese medicine into a readily taught and learnt package. ${ }^{8}$ Modernisation transformed the way Chinese medicine was conceptualised, focusing on clinical practice rather than theory. Moreover, the theory itself was also modified by removing from the curriculum elements that were considered to be rooted in 'superstition' (mixin). Chinese medicine, or TCM, thus became a constructed body of knowledge, more acceptable to the Western medical mind, and more readily assimilated into Western medicine than previous attempts in the seventeenth, eighteenth and nineteenth centuries.

\section{Acupuncture anaesthesia}

The increased interest in acupuncture in Britain (and the rest of Europe) was in part due to its use in analgesia and anaesthesia. The rising status of Chinese medicine in China encouraged more research to be carried out to investigate its therapeutic effects, especially that

\footnotetext{
${ }^{6}$ Mao Zedong 1958, as quoted in Lo 2005B, p. 9.

7 Scheid 2002, p. 65.

${ }^{8}$ See Scheid 2002 and Taylor 2000 for details on the construction of TCM in China in the twentieth century.
} 
of acupuncture, and in the 1950s, the phenomenon of acupuncture anaesthesia was 'discovered'. In Britain, news of the first operation in China using acupuncture anaesthesia arrived in 1958. The technique was first used to relieve post-tonsillectomy pain at the First People's Hospital in Shanghai. Extended to pain relief during other surgery, it was then applied as a form of analgesia for tonsillectomies. In 1971 and 1972, groups of American and British medical men toured medical facilities in China and reported many cases of acupuncture anaesthesia in the Fournal of American Medical Association (JAMA) and British Medical Journal (BMF) with great enthusiasm, stating that they were 'astounded by the use of acupuncture anaesthesia in major surgery'. ${ }^{9}$ Soon, interests were aroused and dramatic stories emerged in almost every issue of the BMJ and Lancet of British doctors who had witnessed open-chest thoracic operations performed with no chemical anaesthesia but a few vibrating needles, where the patient remained alert and cooperated with the surgeon throughout, and after, walked out of the operating theatre with a wave. However, not all members of the medical community were so accepting of this new phenomenon. Heated debates arose between the witnesses who encouraged further investigation into the method, and the sceptics who pronounced acupuncture anaesthetic to be 'effective use of hypnosis', dependent on the susceptibility of the patient and the persuasion of the acupuncturist. ${ }^{10}$

As medical interest in acupuncture increased during the 1960s and 1970 s as a result of acupuncture anaesthesia, lay interest was also aroused by the popular press in both Europe and the USA when in 1971, American reporter James Reston who accompanied President Nixon on his visit to China, published his successful encounter with acupuncture in the New York Times. ${ }^{.1}$ Also on the trip was President Nixon's personal physician, Dr Walter Tkach, who wrote a favourable report on his return to the United States, entitled 'I Have Seen Acupuncture Work'. ${ }^{12}$ These accounts were widely read and discussed, evoking a flurry of interest amongst academic establishments as well as helping to introduce and popularise acupuncture (albeit only in

${ }^{9}$ Dimond 1971, pp. 1558-63; and Hamilton et al. 1972, p. 352.

${ }^{10}$ Wall 1972, p. 129, as quoted in Macintosh 1973, p. 455.

1 Reston 1971, p. 1.

12 Tkach 1972, as cited in Macdonald 1982, p. 105. 
its anaesthetic form) to the lay medical consumers in North America and Europe. However, use of acupuncture for surgical anaesthesia was almost unknown in pre-Maoist China, and its use was limited to only about $10-15$ per cent of all surgical operations done in major hospitals throughout China. ${ }^{13}$ Reports on acupuncture anaesthesia therefore began to taper off rapidly after the fall of the Gang of Four in the late 1970s; and speculation soon emerged in the West that the technique had been promoted (and exaggerated) because of the Chinese nationalist desire to demonstrate the superiority of Chinese tradition in the face of Western scientific scrutiny: it was a 'political hoax'. ${ }^{14}$ Despite this set-back, interest in acupuncture did not suffer, either in China or Europe; on the contrary, the international hype of acupuncture anaesthesia gave untrained doctors in Europe the necessary impetus to study acupuncture in a scientific manner; while in China, the Chinese government encouraged further clinical research, which took place from 1950 to 1990 .

\section{Evaluation}

In researching acupuncture, it soon became clear the problems inherent in attempting to apply western evaluative methods to traditional practices such as acupuncture. Whether to use single- or doubleblind trials, how it should be controlled and what type of placeboif any - should be used, are only some of the barriers in designing effective and fair acupuncture trials. Such problems are difficult to solve because of the individualistic way in which acupuncture is practiced. For example, the acu-points used to relieve a certain symptom vary between different patients and different practitioners, so it seems almost impossible to devise a trial using a 'standardised' set of acupoints when there exists no such concept. Placebos are also the topic of heated debates: obviously it is unethical and ineffective to have a no-treatment control group, and many methods of control have been theoretically considered to isolate the elements of true acupuncture, including mock transcutaneous electrical nerve stimulation (TENS) and minimal acupuncture, where the patient is needled superficially at sites not associated with classical acu-points, which is a favoured

${ }_{13}$ Smith 1974, pp. 367-70.

14 Skrabanek 1981, p. 481; see also Lo 2005C, p. 11. 
option. But too little is known about the effects of acupuncture to dismiss entirely that needling superficially at non-acu-point sites would produce no effects, or effects that do not interfere with the elements of true acupuncture.

Thus, in attempting to evaluate acupuncture, the technique will inevitably need to become standardised and modernised in one way or another, against its empirical nature. The search for repeatable acupuncture prescriptions, regardless of context, is invaluable for its continued use and assimilation into western medical practice, especially in the hospital setting, because it renders the practice 'scientific'. In this case, the loss of tradition is deemed a worthy sacrifice. Moreover, the accreditation of only those elements of acupuncture that are consistent with realities defined under the conditions and confinements of western biomedical trial means that much more of acupuncture is lost than its tradition. Under the scrutiny of scientific evaluation, it becomes medicalised in a process not unlike completing a jigsaw puzzle using scissors. But, having said this, conforming to clinical evaluation legitimises the practice in the eyes of the Western medical community, promoting safety of practice and standardisation of training and treatment, allowing acupuncture to gain the status of a 'Professionally Organised Alternative Therapy' in the House of Lords report in $2000 .^{15}$

\section{Alternative Medicine}

Mao's Cultural Revolution of 1966-1976 closed China to the West, only to be re-opened after his death and under the leadership of Premier Deng Xiaoping in the 1980s. In subsequent years, many British medical men went to China (and Hong Kong) to study and experience acupuncture. Moreover, emigration to Britain, too, increased dramatically during this period. Among the opportunity-seekers were Chinese medical practitioners of both western and traditional medicine. Without a system of regulation or registration at the time, lay acupuncture began to thrive with the setting up of numerous highstreet 'shops' offering Chinese medicine and acupuncture. These pioneering Chinese acupuncturists had visualised a niche in the British

${ }^{15}$ House of Lords Select Committee on Science and Technology's Sixth Report 2000, Chapter 2, Box 1. 
medical market for alternative medicine: a fashionable trend that began in the 1960s and peaked in the 1980s, reflecting public concern with the 'rise of scientific medicine and the fall of the patient'. ${ }^{16}$ Increasingly, accidents such as the Thalidomide incident instilled a degree of doubt and suspicion in the public's faith in biochemical medicine and alternative practitioners darted to meet these concerns. Biomedicine viewed the body mechanically, 'concentrating on smaller and smaller fragments of the body... los[ing] sight of the patient as a whole being, ${ }^{17}$ whereas the classical theory of acupuncture prominently featured the concept of $q i$, which practitioners used in the creation of a new rhetoric of acupuncture that centred on holism. The individualistic and holistic approach emphasised patient consultation and a personal diagnosis, features that were becoming lost in western biomedicine, and thus drawing a large number of the population to the alternative practitioners of acupuncture. Through the creation and occupation of this niche in the medical market, and the establishment of clinical scientific evaluation at the same time, acupuncture began a process of incipient professionalisation.

\section{Education and training}

A key feature of professionalisation is the establishment of standardised education and training. As acupuncture became institutionalised and standardised in China as an integral part of the constructed TCM package, which was more assimilable, and with the opening up of China later in the twentieth century, education and training in acupuncture in Britain became a feasible development. In as early as the 1960s, with increased interest in and practice of acupuncture, colleges were set up to develop a new generation of acupuncturists in the West. This process began with J. R. Worsley, who established the College of Traditional Acupuncture (CTA) in 1966, and in 1972 the International College of Oriental Medicine (ICOM) was founded by Dr J. D. Van Buren 'as a direct result of many students wishing to learn acupuncture from an acknowledged master of this fascinating subject'. ${ }^{18}$ In Britain today, there are eight major accredited institutions

${ }^{16}$ Buckman and Sabbagh 1993, p. 25.

17 Prince Charles 1986, as quoted in $B M A$ Report 1986, p. 1.

${ }_{18}$ International College of Oriental Medicine: http://www.orientalmed.ac.uk/ college/history.htm 
teaching acupuncture, mostly founded in the early to mid-1990s when acupuncture reached a peak of interest through the alternative health movement; and two more are in the accreditation process. ${ }^{19}$ These institutions have each contributed to the rise of lay acupuncture so that today there are over 2,500 practitioners registered with the British Acupuncture Council alone; a figure which is difficult to ignore when one considers whether acupuncture has become a professional body. ${ }^{20}$

\section{'Western style' acupuncture}

In addition to the rise of lay acupuncture in the later decades of the twentieth century, a new phenomenon of 'western style' or 'medical' acupuncture began to appear in the medical market, created by physician Felix Mann who proposed that meridians and acupoints do not exist, but acupuncture still works. His technique removed all elements of tradition from acupuncture, so that the selection of points is based mainly, but not exclusively, on the dermatomal distribution of pain rather than the traditional distribution of points along the meridian. Mann eventually organised annual meetings for all those he had taught, which culminated in the founding of the British Medical Acupuncture Society (BMAS) in 1980. ${ }^{21}$ Today, BMAS members are mostly general practitioners, nurses and physiotherapists who, under great patient demand, take part-time courses in medical acupuncture in order to offer it as an adjunct to their orthodox treatments.

There are both positive and negative aspects to the creation of this new form of acupuncture. On the one hand, it makes acupuncture 'respectable' as a biomedical modality so that it can be accepted by orthodox members of the British medical community, such as

19 The accreditation process is discussed later in the chapter (see 'The Beginnings of Professionalisation'); the eight fully accredited institutions are the College of Integrated Chinese Medicine (CICM) in Reading; College of Traditional Acupuncture (CTA) in Leamington Spa; International College of Oriental Medicine (ICOM) in Sussex; London College of Traditional Acupuncture and Oriental Medicine (LCTA); Northern College of Acupuncture in York; School of Five Element Acupuncture (SOFEA) in London; University of Westminster (School of Integrated Health) in London; and University of Salford (School of Community, Health Sciences and Social Care) in Greater Manchester.

${ }^{20}$ British Acupuncture Council, August 2004, p. 1.

${ }^{21}$ See Baldry 2005, pp. 2-12. 
general practitioners, who have previously viewed traditional Chinese acupuncture with doubt and hostility. On the downside, the development of medical acupuncture within the ethos of Evidence-Based Medicine and Integrated Medicine means that the original paradigm in which traditional acupuncture is rooted is abandoned in the controlling grip of biomedical science. Moreover, the biomedical paradigm that is imposed on Chinese acupuncture destroys its richness, subtleties and insights, only to be replaced by something mechanistic, materialistic and reductionist, evoking fear from the traditionalists that the rise of medical acupuncture will eventually mean the end of a 2,000-year tradition. ${ }^{22}$ Are integration and professionalisation really worth such a sacrifice?

\section{The beginnings of professionalisation}

In order to assess the process of professionalisation of acupuncture, one needs to first define the term 'profession', using both historical and sociological definitions to give a more complete picture of its characteristics: ${ }^{23}$

- a united group of workers

- discrete body of knowledge over which members have control

- legal monopoly of practice

- self-regulatory in terms of exams and licensing

In terms of unity, acupuncturists were not a homogeneous group in the 1960s and 1970s. However, as the number of lay acupuncturists increased, especially in the 1980s following the opening-up of China, a number of professional bodies and societies developed individually and later came together in 1987 under the auspices of the Council For Acupuncture (CFA: 1980-1995), presenting a more powerful voice for their practice. ${ }^{24}$ For 15 years, the CFA attempted to reduce internal disagreements and coordinated standards of training and

${ }^{22}$ Churchill, Wainwright: http://www.jcm.co.uk/product.php?catID=281\&opener=0196-281\&productID $=137 \& d=5$

${ }^{23}$ See Cooter 2005, and Jones in Scambler and Patrick (eds) 1997, p. 236.

${ }^{24}$ The CFA comprised of the British Acupuncture Association and Register in affiliation with the British College of Acupuncture, the Register of TCM, Traditional Acupuncture Society, Chung San Acupuncture Society and the International Register of Oriental Medicine. 
Codes of Ethics and Practice among the practitioner members of the societies under its representation, adding an element of social or institutional organisation to acupuncture's popular front.

In 1995, the five member groups of the CFA were unified in the creation of the British Acupuncture Council (BAcC), which not only represented the acupuncturists, but also governed them, acting as a single regulatory body. This unification of members and compromise of interests were the first tentative steps toward professionalisation. However, the BAcC is only one of several umbrella bodies, its Register of over 2,500 members being predominantly western lay acupuncturists. The Association of TCM (ATCM), founded in 1994, represents a large majority of the Chinese medical community, although there are other competing societies that represent different sectors of the ever growing number of Chinese pharmacies. It was established to cover the whole TCM system which includes Chinese herbal medicine and Chinese remedial massage as well as acupuncture. The British Medical Acupuncture Society (BMAS) and the Acupuncture Association of Chartered Physiotherapists (AACP) form the third group of major regulatory bodies, both established in the 1980s to represent those who practise Western Medical Acupuncture, which are mostly members of the western medical profession such as GPs and nurses and physiotherapists. It can be seen from their histories that each of these regulatory bodies represent an entirely different group of acupuncturists, each of whom practise a different style of acupuncture, therefore unsurprisingly there exists a significant conflict of interest and opinion among them. The BAcC, BMAS and AACP wish to promote acupuncture (in their various forms), whereas ATCM, as a proponent of TCM, believes that acupuncture is only a part of this complete system and therefore it is TCM that should be promoted, not acupuncture on its own. The attainment of a 'united group of workers' therefore involves a process that acupuncture has yet to complete.

The second characteristic of a profession involves education. The increasing numbers of acupuncture colleges during the late twentieth century offered courses that varied greatly in terms of length, content, and perceived quality in preparing competent practitioners. Not only this, there also existed a large gulf between the courses offered by lay and medical practitioners. The colleges training lay practitioners provided long intensive training in a range of aspects of TCM and fitted acupuncture into this classical theory (for example, the London School of Acupuncture and Oriental Medicine), 
whereas courses offered by BMAS concentrated on setting acupuncture within a biomedical paradigm with little or no reference to classical theory. Such discrepancies were a major barrier to the professionalisation of acupuncture; only by standardising education and training can practitioners have the best chance of acceptance by the medical orthodoxy. As a result, the British Acupuncture Accreditation Board (BAAB) was set up in 1989 by the aspiring profession to 'assure the public of professional standards by looking at how a course or institution prepares its students for competent, safe and ethical practice as professionals of an autonomous therapy'. ${ }^{25}$ The Board worked closely with the new BAcC to establish Guidelines for Acupuncture Education, and accredited only the institutions whose standards of training met the set guidelines. Soon after, formal links of status and validation with universities were established to help instil professional values in students, mirroring the professionalisation of medicine itself in nineteenth-century Britain with the founding of hospital and university-linked medical schools. ${ }^{26}$

Knowledge and experience were gained not only through education and training but also through the creation of a literature, both in the public sphere, increasing awareness and promoting the therapy, and among the practitioners, acting as a forum for the discussion of issues of interest and concern. Thus during the 1970s and 1980s, there was a flood of both groups of literature. A number of journals appeared that focused on TCM generally and acupuncture specifically, for example, the Fournal of Chinese Medicine (1979), intended for both practitioners and the general public; and Acupuncture in Medicine (1981), produced by BMAS for medical acupuncturists.

In this way, it seems that acupuncture has secured not one, but a set of heterogeneous bodies of knowledge that differ between the societies, organisations and courses, each promoting a different kind of acupuncture knowledge. But, if one looks at the long-established British medical education system for comparison, the curriculum, too, differs from one University to the next, and the methodology of teaching also varies greatly, some teaching through 'vertical' themes and some are body system-based. Therefore it can be said that although education is individual to each establishment, the accredi-

${ }^{25}$ Uddin 1995, p. 5.

${ }^{26}$ See Abel-Smith 1964. 
tation system allows a standard to be set so that the public can be sure of acupuncturists being in control of a 'discrete body of knowledge'. Currently in the UK, alternative/complementary medicine is legally autonomous: anyone who wishes to can call themselves an acupuncturist and provide treatment to the public. Regulation is voluntary and because of membership costs, many practitioners remain unregulated and therefore unchecked. This liberal regulatory regime, in comparison to other European countries where alternative medicine is more strictly controlled, has been part of the impetus for the rise of lay acupuncture in this country, especially in the Chinese medical community. ${ }^{27}$ Increasingly, however, the government has looked to their European counterparts and the importance of public safety, to initiate a process of statutory self-regulation.

\section{Acupuncture in the twenty-first century: regulation, professionalisation and integration}

The government's push for regulation

Many different groups have been involved in the regulation debate, and like any legislative process in history, some support it and other oppose it. Among the parties in favour of regulation of acupuncture is the government (Department of Health) who set the process in motion in 2000 with the House of Lord's report that assessed topics such as whether good structures of regulation were in place for public safety; whether an evidence base has been created and research being carried out; whether the practitioners' education and training were adequate for competent practice; and what the prospects were for the provision of CAM treatments by the NHS. The report then expressed the Department of Health's written evidence that the Government encouraged larger professions to follow the osteopaths and chiropractors in gaining statutory self-regulation, which would 'undoubtedly serve their professions well'. ${ }^{28}$ The main criteria for preferring such a route were, first, whether the therapy posed significant risk to the public from poor practice; secondly, whether the therapy

${ }^{27}$ See House of Lords Select Committee on Science and Technology's Sixth Report, 2000, section 5.51.

${ }_{28}^{8} B M A$ Report 1993, section 5.51 . 
had a pre-existing, well-organised voluntary regulatory system and a consensus among its members that statutory regulation was the desired next step; and thirdly, the presence of a credible evidence base to support its claims. ${ }^{29}$ Acupuncture and herbal medicine were considered to comply with the criteria and therefore supported in moves toward Statutory Self-Regulation (SSR). Already, it can be seen that the government was only encouraging CAM therapies, or aspects of them, whose theoretical frameworks were scientifically based; and that incongruence with western scientific knowledge would mean its rejection as a worthy therapy. This would indicate that perhaps the government only wished to support and further the provision of the needling technique of acupuncture, dissociated from its traditional concepts of $q i$, yin and yang, much like the materialistic transmission of acupuncture during the seventeenth to the twentieth centuries.

Safety was obviously a key concern for the government, probably the dominant impetus for regulation, but many other reasons can be deduced for its support for SSR. Firstly, the NHS was, and has been for some time, buckling under financial pressures, thus not being able to offer patients the kind of care and service it was set up to provide in 1948. At a time when resources were so overstretched that the Secretary of State for Health sent patients abroad for treatment, the Government could see that it should take into account the mass demand for CAM and 'make better use of the roughly 10,000 acupuncturists who are available to work in the health service'.$^{30}$ Another key point concerns the costs that could be saved by acupuncture. Needles are extremely inexpensive compared to other vastly expensive diagnostic tools and therapeutic drugs, thus acupuncture could be used as a cost-effective way to reduce the outpatient waiting list, and help to ameliorate the NHS financial crisis. Perhaps also, it was a matter of power, as under current law the government had no control over the increasing use of CAM therapies. Acupuncture 'clinics' were opening along every high-street, enticing many patients away from the NHS, thus threatening the dominant position of modern standard medicine. Therefore, some strategy of control and regulation had to be exerted to keep acupuncture in check. From these, it seems that some of the government's aims were

${ }^{29}$ House of Lords Select Committee on Science and Technology's Sixth Report 2000, Summary section IV.

${ }^{30}$ Treddinick 2002, p. 6. 
wrapped up in political concerns about healthcare provision, especially within the NHS, rather than recognising the merits of acupuncture as a valid system of medicine and the desire to offer it to the British public.

\section{Support from the professional body}

The British Acupuncture Council (BAcC) is another strong proponent for SSR. Its existing work in voluntary regulation of acupuncturists encouraged the House of Lords to recommend it to take a leadership role in bringing together all relevant bodies to define the best path forward for SSR in its 2000 report. The BAcC believed that regulation would offer acupuncturists a 'unique window of opportunity to safeguard [their] values and to support [their] vision of traditional acupuncture in the legislative framework' ${ }^{31}$ Some members, such as Felicity Moir, believed that voluntary regulation had been successful in ensuring educational, training, and practice standards, with over 2,500 members belonging to the BAcC alone. But at the same time, she recognised that there remained many practitioners who were not regulated in any way, posing a serious problem to patient safety. ${ }^{32}$ Professor Ma, a Chinese practitioner and member of the Association of TCM, believed that SSR will control such 'unorthodox' acupuncturists, many of their clinics will be closed down due to under-qualification so that they will be fewer in number, but 'definitely much higher in quality', which will benefit the public at large, thus 'increas[ing] the status of the profession' and help to 'further the future of acupuncture towards understanding and acceptance' ${ }^{33}$ Therefore, SSR was also seen as the next step forward for the future of profession, and as Mike O'Farrell, the Chief Executive Officer of BAcC suggested, it will help to 'step up acupuncture so that it [can be] recognised by the government and the public'. ${ }^{34}$ O'Farrell also conveyed that through gaining credibility, acupuncture will have a hope of becoming more incorporated into the NHS, 'not guaranteed of course, but once regulated, it will make it easier

31 BAcC Executive Committee, 2001, p. 21.

${ }^{32}$ Personal communications with Felicity Moir, 6 April 2006.

33 Personal communications with Professor Ma, 15 March 2006.

${ }^{34}$ Personal communications with Mike O'Farrell, 17 March 2006. 
for the decision makers to understand its role in medicine, and be willing to listen to it'. ${ }^{35}$

Currently, the vast majority of acupuncture treatments are received privately, and predominantly by the urban middle class who can afford such expenses. But if one looks at the provision of acupuncture treatments in China, for example, it is clear that it has such a wide potential, and should therefore be made available to a larger proportion of all social classes of the British population, via the NHS; regulation would make this a possibility. Additionally, SSR could help to increase acupuncture's clientele as medical insurers would be more willing to accept statutorily regulated practitioners.

\section{Opposition to regulation}

Although there was unanimous support from the Government and BAcC Executive Committee (EC) since 2000 for the move towards SSR, practising acupuncturists were more hesitant, with opinions divided between those who agreed with the BAcC EC in the merits of SSR, and those who believed that it would more likely jeopardise the autonomy of the profession. However, in October 2001, a vote was taken among BAcC members and 90 per cent of voting members said 'Yes' to the profession working towards a suitable route for SSR. ${ }^{36}$ Although it may seem that this was an unprecedented unanimity of acupuncturists to compromise and cooperate, it should be made clear that the 90 per cent 'Yes' vote was only out of a 66 per cent turnout, suggesting that far from being undisputed, the decision for SSR was also heavily opposed by many members. ${ }^{37}$ The BAcC Newsletters convey this opposition well, publishing heated discussions over the motives and merits of achieving SSR, many comparing the process with that implemented during the regulation of osteopaths in the late 1990s. Even though osteopathy and acupuncture have very different histories, it is necessary to consider this when comparing the two in regard to SSR.

Another opposing view to SSR was that, although one of its main aims was to guarantee safety and excellence of practice (and thus professionalism) for the wellbeing of the patients, one only has to

\footnotetext{
35 Ibid.

36 MacPherson 2001, p. 2.

37 Uddin 2001, p. 4.
} 
look at incidents (of which there are many), such as the Harold Shipman case, to see the failings of SSR. The regulatory law would only prevent the misuse of the title 'acupuncturist', not malpractice by untrained practitioners. Thus how much validity does the public safety claim really have in the push for SSR of acupuncture? And could perceived benefits for public interest outweigh the deleterious effects such as 'curtailed innovation, higher fees and training costs, restricted entry to the field, profession-centred bias, and the triumph of institutional vested interests'? ${ }^{38}$

\section{The struggle for control}

Practitioners were also concerned about the loss of professional autonomy that may accompany regulation. Acupuncturists have always prided themselves in their independent position and being in control of their own practice; but if SSR is enforced, they will be controlled by an over-arching regulatory body, which may ultimately 'biomedicalise' acupuncture to conform to western scientific thought. This concern was expressed in the antagonism towards the work of the Acupuncture Regulatory Working Group (ARWG), which was set up in 2002 by the Foundation for Integrated Health and the Department of Health, consisting of representatives from the British Acupuncture Council (BAcC), British Medical Acupuncture Society (BMAS), Acupuncture Association of Chartered Physiotherapists (AACP) and British Academy of Western Acupuncture (BAWA). This group was set up to discuss and propose the finer details of how SSR will be implemented, in the best interest of both the profession and the public. Its report introduced many concepts that invoked strong negative responses from the BAcC members, one of which was the thrust towards a 'unified acupuncture profession', as stipulated by the House of Lords report in 2000. First impressions would deem this term to be positive and a step in the right direction towards integration. However, a closer look reveals the cost of such unity. It was acknowledged that Traditional Oriental Acupuncture (TOA), as practised by BAcC members, and Western Medical Acupuncture (WMA), as practised by members of BMAS and AACP, were two distinctive innovations and difficult to amalgamate. Therefore two

38 Baker 2002, p. 19. 
options were suggested for their regulation: first, a register primarily for TOA, with WMA registered by the regulatory bodies of their major occupation, and secondly, two separate registers for TOA and WMA. Both options were rejected by the ARWG because they would not work towards a 'unified acupuncture profession'. Instead it was decided to move towards a set of proposals based on a comprehensive register under a single registering/regulatory body, including establishing equivalences between the training of different styles of acupuncture by the adoption of National Occupational Standards. This seems an impossible task because Traditional Oriental Acupuncture involves undergraduate training, whereas Western Medical Acupuncture is similar to a postgraduate course. Therefore the two cannot be equivalent, and if termed equivalent, it would convey the impression that WMA practitioners, having done a postgraduate course, are more highly qualified professionals in acupuncture than TOA practitioners, which would be a misleading and incorrect concept. The worry here was, therefore, about control. If WMA was to be deemed a more expert body, it would gain control over TOA, whose professional autonomy, and the traditions of their practice, would thereafter be lost in the orthodox western world.

\section{The Chinese medical community}

Another aspect of the debate that came across through the Acupuncture Regulatory Working Group was that it was only represented by associations for western practitioners (such as BAcC and BMAS). There was no representation for the Chinese medical community at all, suggesting that they were sidelined and excluded from influence. When questioned about this, Felicity Moir, a senior member of the BAcC, argued that the Chinese associations were invited to all the relevant meetings and debates, but they either did not show up or did not contribute anything when they did attend. The Chinese community seemed not to know what regulation was, or how it was taking place, and were naïve in thinking that they still had control over acupuncture in Britain. ${ }^{39}$ However, the ATCM, through its journal, has kept its members thoroughly up-to-date about the regulation process of both acupuncture and herbal medicine, forming a response

${ }^{39}$ Personal communications with Felicity Moir, 6 April 2006. 
to the Department of Health that they fully supported the regulation of both professions.

Additionally, the response emphasised that the practice of herbal medicine and acupuncture were intimately connected parts of the tradition of Chinese medicine, and it [was] essential that the integrity of this tradition [was] recognised within the statutory framework'. ${ }^{40}$ ATCM therefore strongly supported the proposal for a single overarching regulatory body for CAM, which would allow the registration of acupuncturists and herbalists under single discipline titles, encourage inter-disciplinary working and, in particular, allow for registration of TCM practitioners, who are qualified in both Chinese herbal medicine and acupuncture. This form of registration would give due recognition to TCM, and avoid the need for TCM practitioners to register with two separate statutory bodies, ensuring the highest levels of participation in the regulatory process. ${ }^{41}$ When interviewing Professor $\mathrm{Ma}$ and Wei Hong Tang, it became extremely clear why the Chinese medical community (as represented by ATCM) had largely neglected the acupuncture regulatory process. Both practitioners were trained in TCM in China as a complete system of medicine involving many techniques, including acupuncture, herbal medicine and therapeutic massage. The techniques were not meant to be used independently, but as a part of the whole TCM innovation. Therefore, since coming to the UK to practise, they continued viewing acupuncture, not as a profession on its own, but an integral part of TCM. ${ }^{42}$ ATCM has thus fought for the recognition of this crucial aspect of Chinese medicine that has been hugely neglected by the western world. While the Association accepted those who wished to set acupuncture apart from TGM (that is, all 'acupuncturists'), or introduce different acupuncture styles (such as western acupuncture), they insisted that Chinese practitioners should be allowed to maintain a strong tradition of a complete system. They have, therefore, not participated in the discussion for the regulation of acupuncture alone, because simply to them, there is no acupuncture without herbal medicine and other aspects of TCM. This viewpoint is particularly different from that of the British Acupuncture Council,

${ }^{40}$ Alliance of Professional Associations of Chinese Medicine (Register of Chinese Herbal Medicine and ATCM) 2004, p. 31.

${ }^{41}$ Ibid.

42 Personal communications with Professor Ma, 15 March 2006; and Wei Hong Tang, 6 April 2006. 
who clearly considered acupuncture a viable and independent profession, thus rejecting the Department of Health's proposal for a CAM Council. Instead, a General Acupuncture Council was the preferred model, which would 'provide the best guarantee of the preservation and enhancement of the educational standards ... and would... have greater direct influence and control over all users of the acupuncture techniques, not just the acupuncturists on its register'. ${ }^{43}$ The government had a raging battle on its hands, as ATCM and BAcC disputed internally, and inter-professionally with each other. Both viewed their professions differently and therefore requested different outcomes from the SSR process. The Department of Health has since agreed to a tripartite system of regulation of Acupuncturists, Herbalists, and Traditional Chinese Medicine Practitioners (who practise TCM that involves both acupuncture and herbal medicine) as a means of compromise between all the representative bodies.

\section{Conclusion}

Despite the strong push towards regulation from the Government, the subject remains the topic of heated debates. Those against it believe that regulation will destroy autonomy and remove the traditional roots of acupuncture, whereas proponents of SSR believe that it is a step in the right direction for the 'profession' and a significant stimulant for integration. However, at the heart of this argument lies the fact that acupuncture has become such a diverse practice in Britain that its practitioners cannot agree on the fundamental question of what acupuncture is. To lay western acupuncturists, such as the BAcG members, 'acupuncture' is a traditional holistic practice involving the use of needles. To the medical acupuncturists, it is the application of needles for nerve and musculo-skeletal stimulation. To the Chinese medical practitioners, 'acupuncture' simply does not exist as an independent form of treatment, but rather a part of the complete system of TCM. Thus, if 'acupuncture' cannot even be defined specifically and unanimously, there can be no acupuncture profession, without which the prospect of integration into British medical culture is still far in the future.

${ }^{43}$ British Acupuncture Council 2004, p. 5; see also, Department of Health 2004. 
However, in Britain today the practice has modernised beyond recognition, and it is in part due to this modernisation of old traditions that the British public has, over the last 30 or 40 years, become increasingly broad-minded and accepting of alternative therapies, especially acupuncture. Especially since the emergence of the World Wide Web, which empowers patients with a mass of information and new choices, acupuncture is no longer a last resort, but at the forefront of the treatment of many conditions, particular functional disorders, for which biomedical science has the least to offer. Therefore in this respect, acupuncture has integrated socially and culturally into the British medical culture, if not politically and scientifically.

This contemporary story of acupuncture has highlighted not only the complexities of assimilation, professionalisation and integration of alternative medicine into mainstream western medicine, but it has also brought to light the difficulties inherent in writing the history of alternative medicine, especially those traditional practices rooted in cultures with which we are unfamiliar. Integration of alternative medicine into western medicine is not a fact, but an idea, and historians should not only look at how it has happened but whether it should happen. The western medical paradigm, and more significantly its institutions, are extremely powerful, and acupuncture seems to be a small fish in the big pond of biomedical science. Thus, if integration is to take place, acupuncture is likely to be changed and 'biomedicalised' in the process, losing everything that made it 'alternative' in the first instance. Does this mean that even if integration is successful, it is in fact a hidden failure? Where does the idea that alternative medicine should not integrate leave its attempted assimilation into western medicine?

\section{References}

Primary Sources

Abdulla, S. 1998, 'Jury Still out on Aspects of Acupuncture', Lancet, 351: 962.

Acupuncture Association of Chartered Physiotherapists: http://www.aacp.uk.com/, URL consulted on 13 March 2006.

Acupuncture Regulation Working Group 2003, The Report of Acupuncture Regulation Working Group: The statutory regulation of the acupuncture profession.

Alliance of Professional Associations of Chinese Medicine (Register of Chinese Herbal Medicine and ATCM) 2004, 'TCM Legislation', Joumal of the Association of Traditional Chinese Medicine, January: 31.

Asian Medicine: Tradition and Modernity, 2005, 1.1. 
Association of Traditional Chinese Medicine (UK), Regulation of Herbal Medicine and Acupuncture -Briefing Paper: http://atcm.co.uk/AppForm/DLA-Briefing.doc, URL consulted on 16 March 2006.

Baker, K. 2002, 'Letters', British Acupuncture Council Newes, April: 19.

Baldry, P. 2005, 'The Integration of Acupuncture within Medicine in the UKthe British Medical Acupuncture Society's 25th Anniversary', Acupuncture in Medicine, 23, 1: 2-12.

Bowers, J. Z. 1978, 'Reception of Acupuncture by the Scientific Community: From scorn to a degree of interest', Comparative Medicine East and West, 6, 2: 89-96.

British Acupuncture Council 2004, Register of Practitioner Members.

- Response to the Department of Health Consultation Document on the Regulation of Herbal Medicine and Acupuncture.

British Acupuncture Council Executive Committee 2001, 'Regulating Acupuncture: Exploring the options', British Acupuncture Council Newes, July: 20-3.

British Acupuncture Council: http://www.acupuncture.org.uk, URL consulted on 12 February 2006.

British Medical Acupuncture Society: http://www.medical-acupuncture.co.uk/, URL consulted on 12 February 2006.

British Medical Association 1986, BMA Board of Science Working Party on Alternative Therapy Report.

— 1993, Complementary Medicine: New approaches to good practice, Oxford and New York: Oxford University Press.

Carrubba, R. W. and J. Z. Bowers 1974, 'The Western World's First Detailed Treatise on Acupuncture: Willem Ten Rhijne's De acupunctura', Journal of History of Medicine and Allied Sciences, 29, 4: 371-98.

Carson, F. 2001, 'Letters', British Acupuncture Council News, June: 19-20.

Caspi, O., T. Woolfson and P. Morrell 2001, 'Integrated Medicine: Orthodox meets alternative', BM7, 322: 168-9.

Churchill, Wainwright: http://www.jcm.co.uk/product.php?catID=281\&opener=0-196281\&productID=137\&d=5, URL consulted on 20 March 2006.

College of Integrated Chinese Medicine (CICM): http://www.cicm.org.uk/, URL consulted on 13 February 2006.

College of Traditional Acupuncture (CTA): http://www.acupuncture-coll.ac.uk/, URL consulted on 13 February 2006.

Department of Health 2004, Regulation of Herbal Medicine for Acupuncture: Proposals for statutory regulation.

Dimond, E. G. 1971, 'Acupuncture Anaesthesia: Western medicine and Chinese Traditional Medicine', $7 A M A, 218,10$ : 1558-63.

Dowie, S. 2003, 'Pillow Needles and C Scores as reflections on growing as Acupuncturist', EJOM, 4: 22-4.

Fisher, P. and A. Ward 1994 'Medicine in Europe: Complementary medicine in Europe', BMF, 309: 107-11.

Hamilton, S., P. Brown, M. Hollington, and K. Rutherford 1972, 'Anaesthesia by Acupuncture', BMF, 3: 352 .

House of Lords Select Committee on Science and Technology's Sixth Report 2000, 'Complementary and Alternative Medicine': http://www.publications.parliament.uk/ pa/ld199900/ldselect/ldsctech/123/12301.htm, URL consulted on 11 February 2006.

International College of Oriental Medicine (ICOM): http://www.orientalmed.ac.uk/, URL consulted on 13 February 2006.

Kaempfer, E. 1996, Exotic Pleasures: Fascicles III curious scientific and medical observations, translated by R. W. Carruba, Southern Illinois: University Press.

London College of Traditional Acupuncture and Oriental Medicine (LCTA): http://www.lcta.com/, URL consulted on 13 February 2006.

Macintosh, R. 1973, 'Tests of Acupuncture', BM7, 3: 454-5. 
MacPherson, H. 2001, 'A Mandate for Suitable Route to SSR, but where to go from here?', British Acupuncture Council News, November: 2.

Northern College of Acupuncture: http://www.chinese-medicine.co.uk/, URL consulted on 13 February 2006.

Reston, J. 1971, 'Now, About My Operation in Peking; Now, let me tell you about my appendectomy in Peking ...', New York Times, 26 July 1971.

School of Five Element Acupuncture (SOFEA): http://www.sofea.co.uk/, URL consulted on 13 February 2006.

Skrabanek, P. 1981, 'Endorphins Through The Eye of a Needle (Editorial)', Lancet, 1: $480-2$.

Smith, A. J. 1974, 'Medicine in China: Best of the old and the new', in BMF, 2: $367-70$.

Treddinick, D. 2002, 'David Treddinick in Parliamentary debate January 2002', British Acupuncture Council Newes, February: 4-8.

Uddin, J. 1995, 'Editorial', European Fournal of Oriental Medicine, 6: 5.

— 2001, 'On the 6th October...', British Acupuncture Council News, November: 4.

University of Salford (School of Community, Health Sciences and Social Care): http://www.chssc.salford.ac.uk/, URL consulted on 13 February 2006.

University of Westminster (School of Integrated Health): http://www.wmin.ac.uk/sih/, URL consulted on 13 February 2006.

Wen, D. 2005, 'The Drum of Chinese Medicine's Modernization Beats Loudly', Acupuncture Today, 06: www.acupuncturetoday.com, URL consulted on 25 March 2006.

White, C. 2004, 'Regulatory Body Proposed for Acupuncturists and Herbalists', $B M 7$, 328: 604 .

\section{Interviewes}

Professor Ma

Alicia Grant

Mike O'Farrell

Felicity Moir

Wei Hong Tang

Dr Wendy Knowles Ahmad
15 March 2006

15 March 2006

17 March 2006

06 April 2006

06 April 2006

08 April 2006

\section{Secondary Sources}

Abel-Smith, B. 1964, The Hospitals, 1800-1948: A study in social administration in England and Wales, London: Heinemann.

Alter, J. S. (ed.) 2005, Asian Medicine and Globalization, Philadelphia: University of Pennsylvania Press.

Atherton, D. J. 2002, 'An Orthodox Practitioner's View of Chinese Medicine', in K. Chan and H. Lee (eds.), The Way Fonward For Chinese Medicine, London and New York: Taylor \& Francis, 397-413.

Bivins, R. E. 2000, Acupuncture, Expertise and Cross-Cultural Medicine, Basingstoke and New York: Palgrave, in association with Centre for the History of Science, Technology and Medicine, University of Manchester.

Buckman, R. and K. Sabbagh 1993, Magic or Medicine? An investigation of healing and healers, London: Macmillan.

Cook, H. J. 1994, Trials of an Ordinary Doctor: Foannes Groenevelt in seventeenth-century London, Baltimore: Johns Hopkins University Press.

Cooter, R. 2006, The Rise of Modern Medicine 1650-1900 Lecture 8 notes: Professionalization II.

Eckman, P. 1996, In the Footsteps of the Yellow Emperor: Tracing the history of traditional acupuncture, San Francisco: Cypress. 
Jones, I. R. 1982, 'Health Professions', in G. Scambler and D. L. Patrick (eds.), Sociology as Applied to Medicine, London: Bailliere Tindall, 235-47.

Lo, V. 2005A, Asian Medical History Online Lecture Preparatory Notes-Lecture 2: Diagnosis and Treatment: http://www.ucl.ac.uk/histmed/PDFS/Teaching/BSc/C123/C123Lecture2.pdf, URL consulted on 23 February 2006.

2005B, Asian Medical History Online Lecture Preparatory Notes-Lecture 8: TCMThe Construction of Tradition: http://www.ucl.ac.uk/histmed/PDFS/Teaching/BSc/ C123/C123-Lecture8.pdf, URL consulted on 26 February 2006.

2005C, Medical History Online Lecture Preparatory Notes - Lecture 10: Transmission of Asian Medicine to Europe: http://www.ucl.ac.uk/histmed/PDFS/Teaching/BSc/C123/ C123-Lecture 10a.pdf, URL consulted on 1 March 2006.

Lo, V. and V. Scheid 2005, 2,000 Years of Chinese Acupuncture, London: UCL, with the support of the Wellcome Trust Centre for the History of Medicine at UCL.

Ma, Bo-Ying and A. Grant 2001, 'The Transmission of Traditional Chinese Medicine (TCM) to England (Outline)', in A. K. L. Chan, G. K. Clancey and Hui-chieh Loy (eds.), Historical Perspectives on East Asian Science, Technology and Medicine, Singapore: Singapore University Press, World Scientific, 214-21.

Macdonald, A. 1982, Acupuncture: From ancient art to modern medicine, London and Boston: Allen \& Unwin.

Porter, R. 1997, The Greatest Benefit To Mankind: A medical history of humanity from antiquity to the present, London: HarperCollins.

Scheid, V. 2002, Chinese Medicine in Contemporary China: Plurality and Synthesis, Durham, $\mathrm{NC}$ and London: Duke University Press.

Taylor, K. 2000, 'Medicine of Revolution: Chinese medicine in early communist China (1945-1963)', unpublished Ph.D. thesis, University of Cambridge. 